\title{
Rorschach e sofrimento psíquico grave: funcionamento psíquico nas primeiras crises psicóticas
}

\author{
Rorschach and severe psychic suffering: Psychic \\ functioning in first psychotic crisis
}

\author{
Hayanna Carvalho Santos Ribeiro SILVA \\ lleno Izídio da COSTA²
}

\section{Resumo}

\begin{abstract}
Trata-se de um estudo sobre a estrutura e a dinâmica de personalidade de pessoas em primeira crise do tipo psicótica, questionando criticamente as práticas tradicionais da intervenção precoce. Dez clientes atendidos na esfera individual e familiar pelo Grupo de Intervenção Precoce nas Primeiras Crises do Tipo Psicótico foram submetidos ao método de Rorschach no Sistema Compreensivo. Dados foram comparados com estatísticas descritivas de pacientes esquizofrênicos e adultos não pacientes; a análise enfocou os índices de transtorno do pensamento, traços depressivos, inabilidade social e um grupo de variáveis de relevância específica da população estudada. Resultados indicam que pessoas em primeiras crises do tipo psicótico não possuem traços significativos de transtorno de pensamento. Têm percepções acuradas e incomuns da realidade, sobrecarga afetiva e diminuídas habilidades sociais e dispõem de recursos cognitivos e afetivos para enfrentamento a serem aprimorados. A intervenção precoce nas psicoses pode embasar suas práticas nas características específicas dessa população.
\end{abstract}

Palavras-chave: Intervenção precoce; Teste de Rorschach; Transtornos psicótico.

\begin{abstract}
This is a study about structure and dynamics of the personality of 10 clients in first psychotic-like crises, critically questioning traditional practices in early intervention. Ten clients under individual and family treatment in Grupo de Intervenção Precoce nas Primeiras Crises do Tipo Psicótico were assessed with the Rorschach Inkblot Method in Comprehensive System. Data were compared with international descriptive statistics of schizophrenic inpatients and nonpatient adults, analysis focused the following indexes: Perceptive-thinking, depression, coping and a group of specifically relevant variables for the studied population. Results indicate that individuals in first psychotic-like crises do not have moderate signs of thinking disorder, but accurate and unusual perceptions; affective overload; and diminished social abilities. Such individuals have cognitive and affective resources to be improved. Early intervention in psychosis may adapt practices on the specific characteristics of this population.
\end{abstract}

Keywords: Early intervention; Rorschach test; Psychotic disorders.

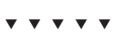

${ }^{1}$ Universidade de Brasília, Instituto de Psicologia, Grupo de Intervenção Precoce nas Primeiras Crises do Tipo Psicótico. Brasília, DF, Brasil.

2 Universidade de Brasília, Instituto de Psicologia, Programa de Pós-Graduação em Psicologia Clínica e Cultura. Campus Universitário Darcy Ribeiro, Ala Sul, Sala Al-040, Asa Norte, 70910-900, Brasília, DF, Brasil. Correspondência para/Correspondence to: I.I. COSTA.

E-mail: <ileno@unb.br>.

Agradecimentos: à Profa. Elisa Walleska Costa pela colaboração nas análises dos dados. 
A psicose é uma manifestação sindrômica cuja formulação tem sido marcada por confusões, enganos e diversas dificuldades quanto à conceituação (Costa, 2003). A tradição psiquiátrica descreve os transtornos psicóticos como síndromes de transtorno de pensamento sem causação orgânica determinada (American Psychiatric Association [APA], 2000). A principal característica é a perda de contato com a realidade, que levaria a formulações bizarras do juízo e a distorções perceptivas. Os sintomas podem ser categorizados em positivos (delírio, alucinação e fala desorganizada) e negativos (retraimento social e embotamento afetivo); (Dalgalarrondo, 2001; Gabbard, 2006).

$O$ atual Diagnostic and Statistical Manual of Mental Disorders (DSM-IV, Manual Diagnóstico e Estatístico de Transtornos Mentais) (APA, 2000, p.298) organiza os Transtornos do Espectro Psicótico nas seguintes categorias: Esquizofrênico; Esquizofreniforme; Esquizoafetivo; Delirante; Psicótico Breve; Psicótico Compartilhado; Psicótico Devido a uma Condição Médica Geral; Psicótico Induzido por Substância. Sintomas psicóticos também podem estar presentes em transtornos de personalidade, demências e transtornos de humor, entre outros.

O uso tradicional do psicodiagnóstico na psicose tende a desconsiderar as implicações sociais, morais e valorativas da atribuição sistemática do rótulo da doença mental ao indivíduo (Fox \& Prilleltensky, 1997). Segundo Szasz (1971; 1979), crítico da psiquiatria coercitiva, o conceito de doença mental é uma metáfora que o cuidado médico tradicional da loucura toma na literalidade. A esquizofrenia seria, na nosografia psiquiátrica, a principal manifestação psicótica. Costa (2003) argumenta, à luz da filosofia analítica, a "inviabilidade científica do conceito de esquizofrenia enquanto paradigma fundamental das psicoses". Fatores culturais, históricos e ideológicos que constituem o indivíduo são ignorados na formulação tradicional do diagnóstico de doença mental, apesar de serem marca do enlouquecimento, dado que o sujeito se insere em uma realidade que o constrói e que é construída por ele. Para McGorry (2011), o cuidado com a saúde mental não deve aguardar por degringolamentos mais cronificados à espera de um enquadre 338 sintomatológico em determinada categoria. Ao contrário, para esse autor, os primeiros sinais de sofrimento psíquico devem ser adereçados com rapidez e atenção.

A partir dessa perspectiva, a leitura de um quadro psicopatológico não pode considerar os sintomas apenas como manifestação sindrômica individual, uma doença individual. A psicopatologia crítica considera o ser humano para além de seu aparato biológico e refere o sintoma ou queixa como parte integrante de um discurso, inserido em um contexto social (Costa, 2010; Moreira \& Sloan, 2002). Sintomas psíquicos são considerados como uma linguagem substituta, desenvolvida em face de dificuldades no uso ordinário da linguagem compartilhada, centralmente envolvendo fatores familiares e históricos fundamentais (Costa, 2003). São manifestações da disposição fundamental humana, pathos (Martins, 2003), e o objetivo terapêutico não deve ser sua extinção, como ocorre com o diagnóstico rígido e o uso indiscriminado de psicofármacos. Por vezes, a medicação psiquiátrica reflete a promessa de eliminar sofrimentos existenciais (Costa, 2010). Na psicopatologia crítica, ao contrário, manifestações páthicas demandam compreensão em respeito à sua complexidade e contextualização.

Crises do tipo psicótico são conceituadas como episódios em que uma pessoa apresenta sintomas psicóticos (Aitchison, Meehan, \& Murray, 1999). Nas primeiras crises, o indivíduo tem potenciais preservados para superação do sofrimento, ferramentas psíquicas e sociais para enfrentamento do estresse em grau e qualidade mais adaptativos, em comparação com períodos de maior cronificação (Salokangas \& McGlashan, 2008).

Antes mesmo do primeiro episódio psicótico, alguns sintomas podem estar presentes, tais como estranhamento quanto ao mundo e a si mesmo, alterações funcionais e relacionais importantes, mas que ainda não são psicóticas. Semelhantes sintomas são denominados pródromos (do grego: aquilo que antecede um evento); (Carvalho, 2006) e indicam fase de alto risco para episódio psicótico. São sintomas como abstração reduzida; perda do senso do eu, dos outros ou do mundo; atenção/concentração reduzida; sensibilidade interpessoal aumentada, 
dentre outros. Pesquisadores salientam a importância de intervenção precoce para promoção de saúde mental e prevenção de desenvolvimento crônico do sofrimento psíquico (Costa, 2010; McGorry, 2011; Yung \& McGorry, 1996).

Ainda são poucos os serviços de intervenção precoce nas psicoses. No Brasil, destaca-se o Grupo de Intervenção Precoce nas Primeiras Crises do Tipo Psicótico (GIPSI), fundado em 2001 pelo Prof. lleno Costa, no Instituto de Psicologia da Universidade de Brasília. O GIPSI acolhe e atende clientes em primeiras crises do tipo psicótico, na esfera individual e familiar, a partir de uma perspectiva sistêmica (Palazzoli, Cirillo, Selvini, \& Sorrentino, 2006). A equipe multiprofissional acolhe o indivíduo em crise, sua família e seu contexto psicossocial com a postura básica de que o sintoma psicótico é gerado na e a partir da sociedade em que o sujeito está inserido (Costa, 2010). Pretende-se, por meio do incentivo à pesquisa, o desenvolvimento de um modelo brasileiro de referência na atenção precoce às primeiras crises do tipo psicótico. Além disso, o GIPSI também objetiva transformação da realidade cultural e social em respeito ao sofrimento psíquico grave.

O GIPSI emprega o termo sofrimento psíquico grave (Costa, 2003) a fim de ressaltar que as primeiras crises do tipo psicótico são vivências de angústia essencialmente humanas - que afetam além do biológico -, marcadas pela intensidade, manejo difícil e pelo potencial incapacitante.

O paradigma internacional da intervenção precoce descreve procedimentos essenciais para prevenir novas crises ou recaídas: detecção precoce, intervenção sensível e específica e atenção psicossocial (McGorry \& Edwards, 2002). Na detecção precoce, recomenda-se o uso de instrumentos psicológicos adequados e que possibilitem uma visão psicopatológica crítica. O GIPSI possui uma sistemática complexa de avaliação, com uso de diversos instrumentos individuais, psicossociais e familiares, dentre eles, o método de Rorschach, ferramenta de investigação profunda da dinâmica e funcionamento da personalidade, para além dos sintomas e da visão sindrômica. É um complexo método perceptivo e projetivo que proporciona a assimilação associativa entre os estímulos e os complexos de sensações intrapsíquicos (Teixeira \& Marques, 2009), investigando aspectos saudáveis e comprometidos da personalidade.

Um dos principais trabalhos de sistematização do Rorschach como ferramenta diagnóstica foi feito por John E. Exner (Exner, 2003). São utilizadas duas perspectivas integradas: a) quantitativa (psicométrica), que avalia os dados objetivos com princípios fundamentais de medida, normatizados a partir de largos estudos estatísticos internacionais e locais, com códigos sensíveis e específicos e b) qualitativa (dinâmica), que avalia particularidades e idiossincrasias do sujeito, conforme fundamento teórico psicanalítico, com análise da sequência, do discurso, das elaborações verbais e outros comportamentos (Weiner, 2000).

A integração das duas perspectivas confere ao método de Rorschach, pelo Sistema Compreensivo, dados amplos e significativos acerca da personalidade, com pouca influência de aspectos de sugestionabilidade e outras manipulações deliberadas (Gacono \& Meloy, 1994; Exner, 2003; Groth-Marnat, 2003; Viglione, Perry, \& Meyer, 2003). Apresenta sensibilidade particular para a avaliação de transtornos no pensamento, em especial para primeiras crises, pois se trata de procedimento simples e cujos resultados podem ser obtidos com maior rapidez, quando comparado a outros instrumentos (llonen et al., 1999). Além disso, o método apresenta índices altamente preditivos (Hilsenroth, Fowler, \& Padawer, 1998) e é utilizado eficientemente em diversos países (Hilsenroth, Eudell-Simons, Defife, \& Charnas, 2007; Nascimento, 2010).

No presente trabalho, foi realizada avaliação de clientes atendidos pelo GIPSI em primeiras crises de sofrimento psíquico grave, por meio do método de Rorschach no Sistema Compreensivo. Objetiva-se uma descrição de aspectos dinâmicos e estruturais desses clientes a respeito de componentes da personalidade significativos e específicos dessa população.

O Sistema Compreensivo apresenta constelações, agrupamentos de variáveis com signifi- 
cância estatística e que podem auxiliar na construção de hipóteses diagnósticas. Uma das constelações importantes na consideração das primeiras crises do tipo psicótico é o Perceptual-Thinking Index (PTI, Índice de Percepção-Pensamento, reformulação do Índice de Esquizofrenia [SCZI]). O índice é função dimensional da identificação de distorções cognitivas, transtornos no pensamento e comprometimento no teste de realidade (Hilsenroth et al., 2007). Estudos referem sua eficácia na diferenciação de pacientes com características psicóticas de outros tipos de pacientes (Dao \& Prevatt, 2006; Ilonen et al., 1999; Jorgensen, Andersen, \& Dam, 2000; Klonsky, 2004) ou sujeitos não pacientes (Hilsenroth et al., 1998; Jorgensen et al., 2000), com menor enfoque nas funções psicodiagnósticas clássicas, sindrômicas/sintomatológicas.

Outras constelações importantes são o Coping Deficit Index (CDI, Índice de Déficit no Enfrentamento) e o Depression Index (DEPI, Índice de Depressão). O CDI relaciona-se a dificuldades importantes nas interações sociais, é considerado medida de inabilidade social ou comprometimento no desenvolvimento de defesas sociais (Exner, 2003). Já o DEPI refere-se a aspectos de depressividade e disforia, sinais relacionados a quadros depressivos mais persistentes, e engloba aspectos relacionais, cognitivos e afetivos de disforia, pessimismo e dificuldades no devir (Carlson, Kula, \& Laurent, 1997).

A avaliação psicológica é importante para a intervenção precoce, uma vez que quanto mais se prolonga o período entre a fase prodrômica e o desenvolvimento das primeiras crises psicóticas, maior o comprometimento afetivo, cognitivo e relacional (McGorry \& Edwards, 2002). Assim sendo, o uso do método de Rorschach na avaliação das primeiras crises do tipo psicótico é consistente com reflexões da psicopatologia crítica no sentido metodológico, e possibilita pesquisas com dados qualitativos e amplos em vez de simplesmente psicométricos. Além disso, é possível contribuir para o avanço das pesquisas em intervenção precoce por meio de avaliação adequada e profunda das pri340 meiras crises de sofrimento psíquico grave.

\section{Método}

\section{Participantes}

Foram avaliados 10 clientes em primeiras crises do tipo psicótico, em acompanhamento psicoterápico individual e familiar no GIPSI, no Centro de Atendimento e Estudos Psicológicos do Instituto de Psicologia da Universidade de Brasília (GIPSI/ CAEP/UnB). Os clientes foram encaminhados para acolhimento por profissionais da rede de saúde, por meio de formulário prodrômico próprio do GIPSI e avaliados quanto aos critérios de exclusão, uma vez que são encaminhados para a rede clientes com histórico de uso de drogas, com indisponibilidade para terapia familiar e com possibilidade de causação orgânica para a crise.

\section{Instrumentos}

Os clientes foram avaliados com o método de Rorschach, parte da sistemática de avaliação psicológica do GIPSI, para análise da estrutura e do funcionamento psíquicos, com fins de psicodiagnóstico e planejamento terapêutico.

\section{Procedimentos}

Os protocolos foram codificados segundo o Sistema Compreensivo (Exner, 2003), com supervisão das aplicações e interpretações no grupo. Foram realizadas devolutivas aos terapeutas individuais e/ou familiares, com enfoque nas considerações terapêuticas, recursos saudáveis e fatores de risco.

Os sumários estruturais foram elaborados por meio do software Ror-Scan, Rorschach Interpretative System, v.6.05 (Carracena, 2005). Dados dos sumários foram comparados com tabelas de estatísticas descritivas de adultos não pacientes (Exner, 2003) e pacientes esquizofrênicos internados (Exner, 1999). Dados discrepantes foram assinalados e interpretados de acordo com o método interpretativo psicodinâmico do Sistema Compreensivo (Weiner, 2000). 
Por ser este um trabalho resultante de iniciação científica, foi incluso no projeto maior intitulado "Estruturação de uma proposta de atenção às primeiras crises psíquicas graves a partir do modelo internacional de intervenção precoce nas psicoses", sob responsabilidade de lleno Costa, aprovado pelo Comitê de Ética em Pesquisa com Seres Humanos do Instituto de Ciências Humanas da Universidade de Brasília, em agosto de 2009.

\section{Resultados}

Os dados obtidos a partir dos protocolos de Rorschach foram comparados com as estatísticas descritivas para adultos não pacientes (Exner, 2003) e pacientes esquizofrênicos internados (Exner, 1999). Variáveis discrepantes foram avaliadas quanto a seu significado qualitativo, nos termos da abordagem à experiência do Sistema Compreensivo (Weiner, 2000).

A maioria dos sujeitos não positivou a constelação PTI (positiva se $\geq 3$, média $=1,6$ ), relacionada a transtornos do espectro psicótico (Figura 1). As constelações $C D I$ (positiva se $\geq 4$, média $=3,2$ ) e DEPI (positiva se $\geq 5$, média $=3,8$ ), contudo, apresentam pontuações elevadas.

A Figura 1 detalha as variáveis relacionadas ao PTI, as médias dos sujeitos avaliados e a comparação com dados de não pacientes e pacientes esquizofrênicos. Os clientes GIPSI apresentam dificuldades em julgar convencionalmente os aspectos

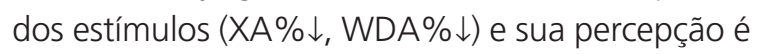
distorcida em grau elevado (X-\% $\uparrow$ ) quando comparados a adultos não pacientes.

Dados indicam que a lógica e a formulação dos pensamentos entre os clientes GIPSI estão preservadas (WSum6 médio = 10,2, Nível $2=1$,4). Ao contrário do que se observa em pacientes esquizofrênicos (média do WSum6 = 44,69), clientes do GIPSI não apresentam grandes prejuízos no julgamento e na elaboração ideativa e cometem falhas mais simples na construção de conceitos (médias clientes GIPSI: WSum6 = 10,2 e Nvl-2 = 1,4). Esse fator indica que as distorções perceptivas $(\mathrm{X}-\% \uparrow)$ encontradas em nossos clientes, as quais são usual-mente relacionadas a um possível trans- torno de pensamento, podem ter mais a ver com à sobrecarga estressora (Nota D, Figura 2).

A maioria dos clientes GIPSI apresenta pontuação nas variáveis da constelação CDI (média do $C D I=3,2$; positiva se $\geq 4$ ), relacionada a dificuldades no trato social e na tolerância ao estresse vivenciado em relacionamentos com o outro (Figura 3). As pessoas com CDI elevado têm diminuída quantidade e qualidade de recursos internos disponíveis para enfrentamento (EA $\downarrow$ ). Os recursos afetivos são

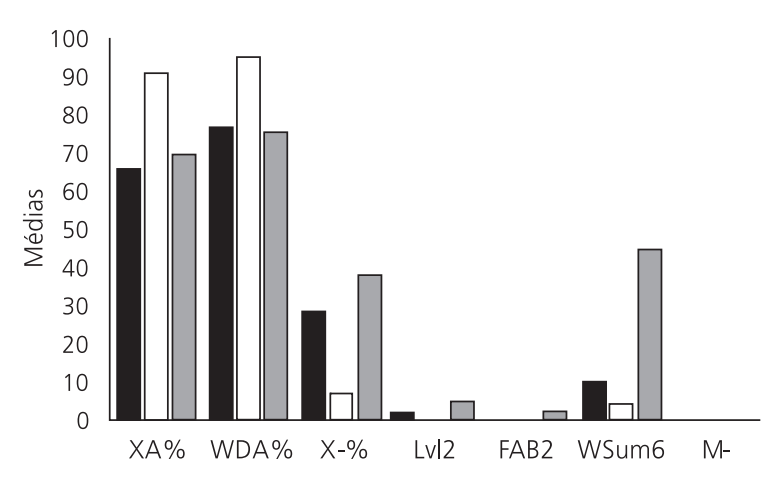

Média GIPSI $\square$ Média não pt. $\square$ Média pt. Esquizofrênicos

Figura 1. Médias das variáveis do PTI, comparação entre os sujeitos GIPSI e as estatísticas descritivas de adultos não pacientes e de pacientes esquizofrênicos.

Nota: PTI: Perceptual-Thinking Index, Índice de Percepção-Pensamento, reformulação do antigo Índice de Esquizofrenia, SCZI; GIPSI: Grupo de Intervenção Precoce nas Primeiras Crises do Tipo Psicótico.

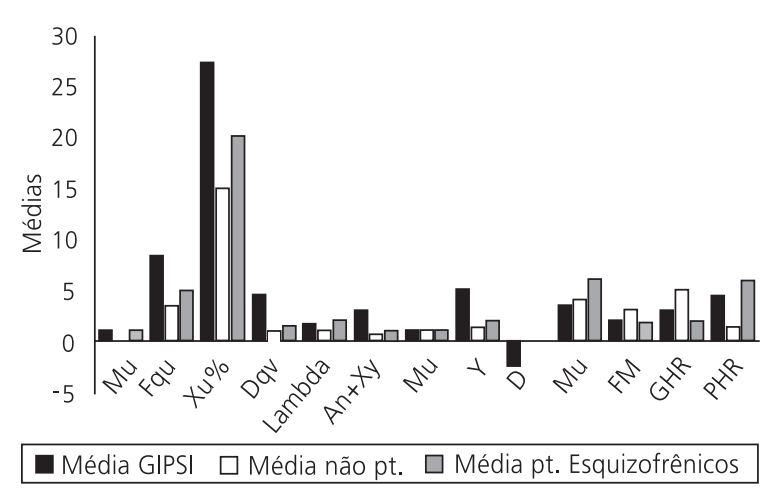

Figura 2. Médias de variáveis com alterações importantes, comparação entre os dados dos sujeitos GIPSI e as estatísticas descritivas de adultos não pacientes e de pacientes esquizofrênicos.

Nota: GIPSI: Grupo de Intervenção Precoce nas Primeiras Crises do Tipo Psicótico. 
particularmente comprometidos, têm número diminuído (WSumC $\downarrow$ ) e são pobremente modulados $(\mathrm{CF}+\mathrm{C}>\mathrm{FC})$.

Fatores que denotam as dificuldades sociais apresentadas pelos clientes GIPSI são: baixa habilidade para perceber aspectos cooperativos nos relacionamentos interpessoais (COP $\downarrow$ ); tendência a assumir um papel mais passivo nas interações, sem grande disponibilidade para iniciar ativamente os comportamentos ("p" $\uparrow$ e "a" $\downarrow$ ).

O Índice de Depressão também apresenta pontuação elevada entre os clientes GIPSI (Figura 4). Os sujeitos têm a demanda afetiva aumentada

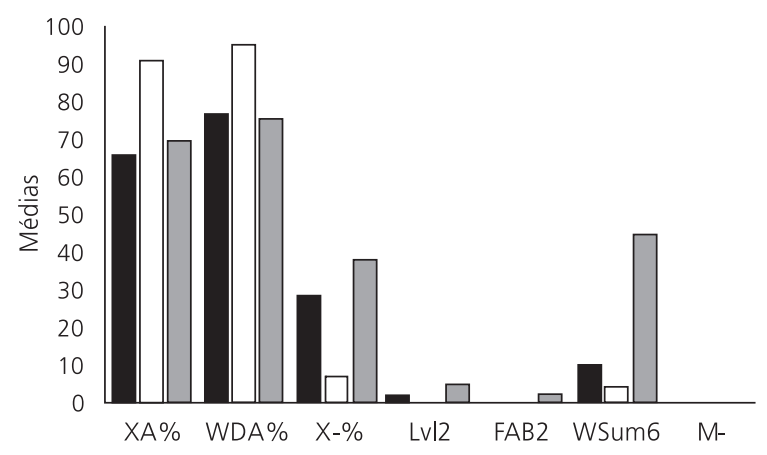

Média GIPSI $\square$ Média não pt. $\square$ Média pt. Esquizofrênicos

Figura 3. Médias das variáveis do CDI, comparação entre os sujeitos GIPSI e as estatísticas descritivas de adultos não pacientes e de pacientes esquizofrênicos.

Nota: CDI: Coping Deficit Index, Índice de Déficit no Enfrentamento; GIPSI: Grupo de Intervenção Precoce nas Primeiras Crises do Tipo Psicótico.

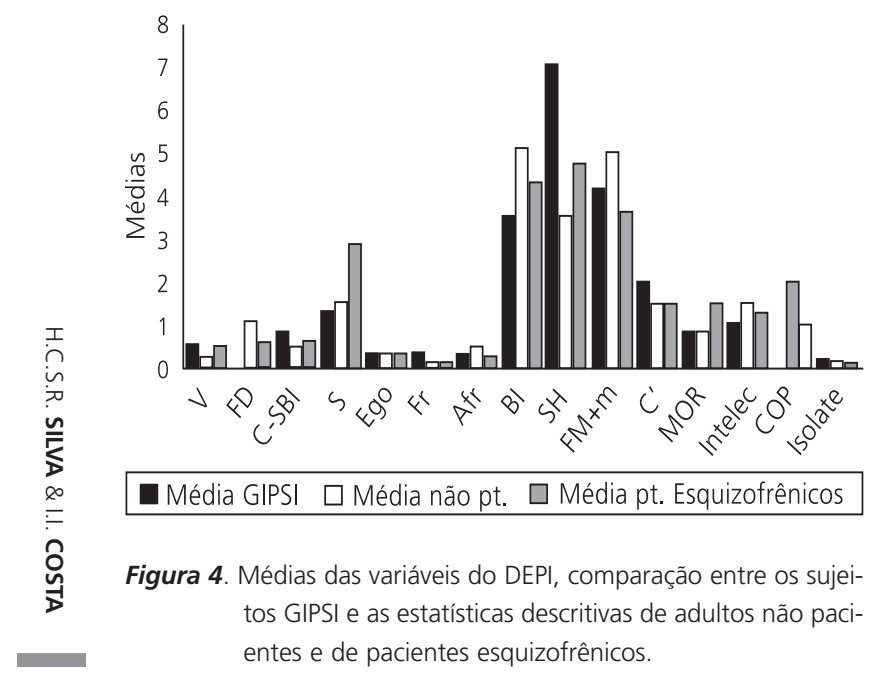

Nota: DEPI: Depression Index, Índice de Depressão; GIPSI: Grupo de Inter342
(SumSH $\uparrow$ ), particularmente relacionada a sentimentos situacionais ou temporários de incapacidade, desamparo, como uma angústia difusa e não simbolizada ( $\mathrm{Y} \uparrow$ ). Tais demandas excedem em muito os recursos para enfrentamento ( $\mathrm{SumSH}>\mathrm{EA}$ ) e podem trazer grandes dificuldades interpessoais, afetivas e cognitivas.

Outro traço presente entre os clientes GIPSI é a ambivalência afetiva, sentimento tanto positivo quanto negativo atribuído ao mesmo tema ou percepto (C-SH Blend $\uparrow$ ). Esse aumento da carga afetiva é característico de situações de crise, e os dados indicam aspecto temporário ou situacional dos fatores estressantes (Nota $D \downarrow$, AdjD dentro da média).

Há um grupo de variáveis (Figura 2), que apresenta alterações importantes, mas que não é contemplado nas constelações anteriores. Dentre essas variáveis, está o aspecto difuso e situacional da angústia que perpassa as demandas afetivas (componente $\mathrm{Y}$ marca a elevação do $\mathrm{SH}$, presente na constelação DEPI). O componente situacional também leva à alteração da Nota D e indica ausência de componentes crônicos nas dificuldades experienciadas pelos clientes GIPSI. A variável relacionada à cronicidade, contemplada na constelação CDI, é a $A d j D$, que se encontra em nível ajustado entre os sujeitos avaliados.

Percebe-se também que, para além de distorções perceptivas, os clientes GIPSI apresentam um aumento nas percepções adequadas, mas muito

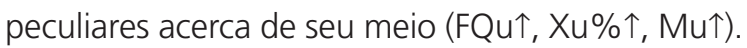
Além disso, há aumento na consideração mais infantil e imatura do meio, com diminuição considerável da atividade de síntese mais sofisticada (DQv^, Lambda个). Há indicadores de temor ou ansiedade em relação ao perigo da integridade do corpo e da

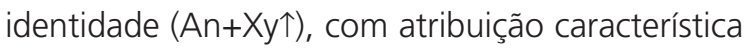
sensivelmente fragilizada da autoestima. Essa dinâmica agrava sentimentos de incapacidade, paralisia e inadequação (também relacionados ao $\mathrm{Y} \uparrow$ ).

\section{Discussão}

Não há indícios suficientes para afirmar comprometimentos severos nas habilidades de me- 
diação dos sujeitos GIPSI, como uma perda de contato com a realidade. A partir dos dados da constelação PTI, subentende-se que os clientes em primeiras crises dispõem de controle ideativo na formulação do pensamento e as distorções estão mais relacionadas a fatores de crise e sobrecarga.

O traço cognitivo de processamento menos sofisticado pode ser um movimento regressivo em relação à atenção e à síntese dos estímulos, resposta aos componentes estressores e de sobrecarga do meio. De fato, a mediação desses clientes é perpassada muito mais por percepções únicas e peculiares do que por distorções perceptivas. $O$ ajuste perceptivo e o teste de realidade permanecem preservados, mas o contato social pode ser dificultado, por se tratar de expressões únicas de percepção da realidade, enfoque maior em aspectos não convencionais do meio.

Essas são características de pessoas criativas e excêntricas, mas podem trazer importantes dificuldades comunicativas caso o meio social seja intolerante à diversidade ou em situações em que a convencionalidade seja uma consideração indispensável.

A passividade encontrada entre os clientes GIPSI pode estar relacionada à dificuldade em pedir ajuda, comum em situações em que se prolonga o período de crise sem oferecimento de acompanhamento adequado. Há um componente de interação mais passiva, que pode comprometer a capacidade criativa na resolução de problemas e tomada de decisão quanto a componentes sociais.

A crise vivenciada pelos clientes avaliados tem importante caráter situacional. A sobrecarga é de dimensão afetiva, e os recursos afetivos não são modulados cognitivamente. A angústia difusa gera potencial para desorganização e impulsividade, sintomas frequentemente relacionados aos estados prodrômicos. Não fosse o excesso de demandas afetivas, os recursos poderiam ser mais bem direcionados para controle, e elaborações cognitivas, mais responsivas à realidade. Apesar de a sobrecarga dos sujeitos ser altamente desorganizadora, não há indícios de distorções ou deslizes muito

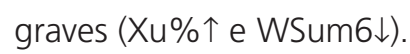

\section{Conclusão}

Por possibilitar avaliação mais abrangente e aprofundada de aspectos saudáveis e psicopatológicos da personalidade, confirma-se a adequação do método de Rorschach como ferramenta de avaliação no paradigma da intervenção precoce e a partir da psicopatologia crítica. Foram desenvolvidos maiores questionamentos quanto ao uso tradicional do diagnóstico de psicoses.

A avaliação de personalidade de pessoas em primeiras crises do tipo psicótico aponta a ausência de componentes graves na formulação de seu pensamento: são pessoas sem indícios válidos de transtorno cognitivo. Semelhantes indivíduos são considerados pela psiquiatria tradicional como psicóticos: um quadro de transtorno de pensamento e perda de contato com a realidade. No entanto, o que se percebe é que esses sujeitos apresentam adequação em suas percepções, mas são excêntricos, não usuais, incomuns. Trata-se de pessoas não convencionais, cuja relação com o meio é perpassada por dificuldades de comunicação e de ajustamento social. Essas considerações referem a uma problemática também do meio, que os considera estranhos e inicia um processo de exclusão sistemática.

Há também fatores situacionais importantes relacionados à crise, os quais referem a aspectos temporários que sobrecarregam os recursos para enfrentamento. Verifica-se que os sujeitos avaliados em primeiras crises não apresentam processo crônico de sofrimento, mas que podem ver-se aliviados das pressões internas e externas por meio do alívio do estresse subjetivo e pelo desenvolvimento de recursos internos.

A avaliação pelo método de Rorschach nas primeiras crises apresenta potencialidades terapêuticas que podem ser tecidas a partir de dados encontrados com rapidez e eficácia. A detecção precoce de um estado prodrômico, a investigação dos recursos internos preservados e o planejamento psicoterapêutico específico e sensível são fundamento da intervenção precoce. O uso do método de Rorschach apresenta-se coerente com essa prática e potencializa seus benefícios. 
O presente estudo fornece dados para compreender dinâmica e funcionalmente sujeitos em primeiras crises do tipo psicótico. Dados for-necem características específicas dessa população, as quais podem subsidiar planejamentos terapêuticos adequados e precoces. Sugere-se fortemente a continuação da presente pesquisa mediante estudos mais aprofundados. Limitações atuais envolvem, por exemplo, a necessidade do uso de tabelas descritivas internacionais por serem ausentes estudos normativos sobre populações psiquiátricas brasileiras.

\section{Referências}

Aitchison, K. J., Meehan, K., \& Murray, R. M. (1999). First-episode psychosis. London: Martin Duritz.

American Psychiatric Association. (2000). Diagnostic and Statistical Manual of Mental Disorders: DSM-IV. Washington, DC: The Author.

Carlson, C., Kula, M., \& Laurent, C. (1997). Rorschach revised DEPI and CDI with inpatient major depressives and borderline personality disorder with major depression: Validity issues. Journal of Clinical Psychology, 53(1), 51-58.

Carracena, P. F. (2005). ROR-SCAN: Rorschach Interpretative System. Versão 6.05. Edmond: Phillip F. Carracena.

Carvalho, N. R. (2006). Primeiras crises psicóticas: identificando pródromos a partir de familiares (Dissertação de mestrado não-publicada). Universidade de Brasília.

Costa, I. I. (2003). Da fala ao sofrimento psíquico grave: ensaios acerca da linguagem ordinária e a clínica familiar da esquizofrenia. Brasília: ABRAFIPP.

Costa, I. I. (2010). Da psicose aos sofrimentos psíquicos graves: caminhos para uma abordagem complexa. Brasília: Kako Editora.

Dalgalarrondo, P. (2001). Psicopatologia e semiologia dos transtornos mentais. Porto Alegre: Artmed.

Dao, T. K., \& Prevatt, F. (2006). A psychometric evaluation of the Rorschach comprehensive system's perceptual thinking index. Journal of Personality Assessment, 86(2), 180-189.

Exner, J. E. (1999). Manual de interpretação de Rorschach: sistema compreensivo. São Paulo: Casa do Psicólogo.

Exner, J. E. (2003). The Rorschach: A comprehensive system. Hoboken: John Wiley \& Sons.

Fox, D., \& Prilleltensky, I. (1997). Critical psychology: An 344 introduction. London: Sage.
Gabbard, G. O. (2006). Psiquiatria psicodinâmica na prática clínica. Porto Alegre: Artmed.

Gacono, C., \& Meloy, R. (1994). The Rorschach assessment of aggressive and psychopathic personalities. Hillsdale: Lawrence Erlbaum.

Groth-Marnat, G. (2003). Handbook of psychological assessment. Hoboken: John Wiley \& Sons.

Hilsenroth, M., Eudell-Simons, E., Defife, J., \& Charnas, J. (2007). The Rorschach Perceptual-Thinking Index (PTI): An examination of reliability, validity and diagnostic efficiency. International Journal of Testing, 7(3), 269-291.

Hilsenroth, M., Fowler, C., \& Padawer, R. (1998). Rorschach Schizophrenia Índex. Journal of Personality Assessment, 70, 514-534.

Ilonen, T., Taiminen, T., Karlsson, H., Lauerma, H., Leinonen, K. M., Wallenius, E., ... Salokangas, R. K. (1999). Diagnostic efficiency of the Rorschach schizophrenia and depression indices in identifying first-episode and severe depression. Psychiatry Research, 87(2-3), 183-192.

Jorgensen, K., Andersen, T. J., \& Dam, H. (2000). Diagnostic efficiency of the Rorschach depression index and the schizophrenia index: A review. Journal of Personality Assessment, 7(3), 258-280.

Klonsky, D. E. (2004). Performance of personality assessment inventory and Rorschach indices of schizophrenia in a public psychiatric hospital. Psychological Services, 1(2), 107-110.

Martins, F. (2003). Psicopathologia II - semiologia clínica: investigação teórico-clínica das síndromes psicopatológicas clássicas. Brasília: ABRAFIPP.

McGorry, P. (2011). International Early Psychosis Association: Presentation. Melbourne: EPPIC early psychosis webinars. Retrieved March 22, 2011, from http://www.eppic.org.au/webinars

McGorry, P. D., \& Edwards, J. (2002). Implementing early intervention in psychosis. A guide to establishing early psychosis services. London: Martin Duritz.

Moreira, V., \& Sloan, T. (2002). Personalidade, ideologia e psicopatologia crítica. São Paulo: Escuta.

Nascimento, R. S. G. F. (2010). Sistema compreensivo do Rorschach: teoria, pesquisa e normas para a população brasileira. São Paulo: Casa do Psicólogo.

Palazzoli, M. S., Cirillo, S., Selvini, M., \& Sorrentino, A. M. (2006). Os jogos psicóticos na família. São Paulo: Summus.

Salokangas, R. K., \& McGlashan, T. H. (2008). Early detection and intervention of psychosis: A review. Nordic Journal Psychiatry, 62(2), pp. 92-105.

Szasz, T. (1971). The manufacture of madness: $A$ comparative study of the inquisition and the mental health movement. London: University Press. 
Szasz, T. (1979). The lying truths of psychiatry. The Journal of Libertarian Studies, 3(2), 121-139.

Teixeira, V., \& Marques, M. E. (2009). O buraco negro na patologia limite: um contributo da/para a técnica de Rorschach. Análise Psicológica, 27(3), 281-293.

Viglione, D. J., Perry, W., \& Meyer, G. (2003). Refinements in the Rorschach ego impairment index incorporating the human representational variable. Journal of Personality Assessment, 81(1), 149-156.
Weiner, I. B. (2000). Princípios de interpretação do Rorschach. São Paulo: Casa do Psicólogo.

Yung, A., \& McGorry, P. (1996). The prodromal phase of first-episode psychosis: Past and current conceptualizations. Schizophrenia Bulletin, 22(2), 353-370.

Recebido em: 25/9/2012

Versão final em: 24/5/2013

Aprovado em: 17/9/2013 
